\title{
The association between habitual physical activity and cigarette cravings, and influence of smokers' characteristics in disadvantaged smokers not ready to quit
}

\author{
M. Haasova ${ }^{1}$ - F. C. Warren ${ }^{1}$. T. Thompson ${ }^{2}$ - M. Ussher $^{3}$ • A. H. Taylor ${ }^{2}$
}

Received: 11 December 2015 / Accepted: 5 May 2016/Published online: 2 June 2016

(C) The Author(s) 2016. This article is published with open access at Springerlink.com

\begin{abstract}
Rationale Habitual physical activity (PA) may have an important role in suppressing cigarette cravings. Systematic reviews show a strong acute effect of bouts of PA on reducing cigarette cravings, and it may be that these effects accumulate.

Objectives The aim was to investigate the relationship between habitual levels of PA and cigarette cravings in disadvantaged smokers not ready to quit by examining baseline cross-sectional data from the Exercise Assisted Reduction then Stop smoking study (EARS).

Methods A series of linear regression models were applied to investigate the relationship between habitual PA and cigarette cravings and to identify additional predictors of cigarette cravings. The analyses were extended by including interaction terms with PA to identify potential moderators of the relationship between PA and cravings.

Results A higher level of moderate intensity PA was associated with lower cravings ( $p=0.033$ ). Additional predictors were the mood and physical symptoms scale $(p=0.007$; higher scores were associated with higher cravings) and alcohol consumption ( $p=0.002$; higher consumption was associated with
\end{abstract}

Electronic supplementary material The online version of this article (doi:10.1007/s00213-016-4326-6) contains supplementary material, which is available to authorized users.

M. Haasova

M.Haasova@exeter.ac.uk

1 University of Exeter Medical School, St. Luke's Campus, Heavitree Road, Exeter EX1 2LU, UK

2 Plymouth University Peninsula Schools of Medicine and Dentistry, Plymouth Science Park, Plymouth PL6 8BX, UK

3 St George's University of London, Cranmer Terrace, London SW17 ORE, UK lower cravings). In addition, a moderation effect of alcohol consumption was found; at higher levels of alcohol consumption, higher PA was significantly associated with higher cravings $(p=0.023)$.

Conclusions Overall, participation in regular PA is associated with reduced cigarette cravings; among those with heavy alcohol consumption, this participation is associated with higher cravings. These exploratory analyses suggest that further research into the relationship between PA, alcohol consumption and cigarette cravings is needed.

Keywords Exercise $\cdot$ Urges to smoke $\cdot$ Cigarette cravings · Cross-sectional $\cdot$ Disadvantaged $\cdot$ Smoking $\cdot$ Alcohol

\section{Introduction}

There is evidence that smokers are less physically active than non-smokers (Kaczynski et al. 2008) and that active smokers are more likely to quit smoking (Marcus et al. 2005). Cravings for cigarettes are a reliable predictor of relapse to smoking (e.g. Killen and Fortmann 1997; Piasecki et al. 2003), brief bouts of physical activity (PA) have a strong acute effect on reducing cigarette cravings (Haasova et al. 2013; 2014; Roberts et al. 2012), and it may be that these effects accumulate across the day. For example, Bloom et al. (2012) reported a negative association between cigarette withdrawal symptoms and weekly PA but only among male psychiatrically hospitalised adolescents, and not females (Bloom et al. 2012). It is important to determine if habitual or chronic PA is associated with both acute and more enduring or stable cravings (Tiffany and Wray 2012) in the general population of smokers and to examine whether these associations vary according to different sub-groups. Such evidence can inform 
the design of PA interventions for smoking cessation (Ussher et al. 2014).

There may be several factors that influence the strength of the relationship between PA and cravings, such as exercise characteristics (e.g. moderate and vigorous exercise has greater acute effects than light intensity; Haasova et al. 2014), socio-demographic factors (e.g. gender differences in motivation for smoking and PA; Bloom et al. 2012), satisfaction from smoking and cigarette dependence. There are also a number of shared correlates of smoking and PA, with lower socioeconomic groups more likely to smoke, drink alcohol and being less active (Bellis et al. 2016). The relationship between PA and alcohol is not simple. Less physically active are more likely to consume alcohol (e.g.Korhonen et al. 2009; Liangpunsakul et al. 2010); however, other studies found the reverse association between PA and drinking (e.g. French et al. 2009a; Kwan et al. 2014; Lisha and Sussman 2010). Mood and measures of emotional well-being are more positive in response to PA (Reed and Ones 2006), and so it is important to control for these factors when assessing the association between PA and cravings. Exploratory work is therefore needed to tease out what factors may moderate the relationship between PA and cravings.

The aim of this study was to investigate the relationship between habitual levels of PA and cigarette cravings in disadvantaged smokers not ready to quit by examining baseline cross-sectional data collected in the pilot randomised trial Exercise Assisted Reduction then Stop smoking study (EARS; Taylor et al. 2014). Additional predictors of cigarette cravings, and potential moderators of the association between habitual levels of PA and cigarette cravings, were also explored.

\section{Methods}

The EARS smoking study (Taylor et al. 2014) was a pragmatic, two-arm pilot randomised controlled trial comparing counselling on PA and smoking reduction (to cut down, then quit), with brief advice on quitting, among disadvantaged smokers who did not wish to quit in the next month. This study examines baseline data for all EARS participants, pooling intervention and control arm baseline data.

\section{Participants}

Eligible participants were as follows: at least 18 years old, smoked at least 10 cigarettes per day for at least 2 years, did not want to quit in the next month, were able to walk continuously for at least $15 \mathrm{~min}$, were registered with a general physician and did not wish to use nicotine replacement therapy to reduce smoking. The EARS study recruited 99 participants between May 2011 and May 2012.

\section{Measures}

Only measures considered in this study are described below; a full list of outcomes is provided elsewhere (Taylor et al. 2014).

\section{Physical activity}

Self-reported PA was assessed by 7-day recall (Blair et al. 1985). Average nightly sleep and minutes of moderate and vigorous intensity PA over the past 7 days were recorded, and minutes of light activity were derived, with light intensity PA including any sedentary time. Minutes of moderate and vigorous intensity PA were then combined (MVPA), and participants were categorised as meeting the national PA guidelines (150 or more minutes of MVPA per week) or not meeting the guidelines (Bull and the Expert Working Groups 2010). In addition, daily energy expenditure (EE; $\mathrm{kcal} / \mathrm{kg}$ ) was calculated from self-reported PA (Blair et al. 1985).

\section{Cigarette cravings}

A 0-5-point Likert scale assessed strength of urge to smoke (SOU; $0=$ no urges to $5=$ extremely strong urges): 'How strong have the urges been to smoke this past week?' (West and Hajek 2004; West and Russell 1985).

\section{Smoking measures}

Self-reported numbers of cigarettes smoked over the past week, smoking satisfaction and psychological reward (10item modified Cigarette Evaluation Questionnaire [mCEQ]; Cappelleri et al. 2007), cigarette dependence (Fagerström Test for Cigarette Dependence [FTCD]; Fagerström 2012; Heatherton et al. 1991) and the age when participants started smoking were collected.

\section{Withdrawal symptoms}

The mood and physical symptoms scale (MPSS) with nine items (restless, irritable, depressed, hungry, poor concentration, poor sleep at night, stressed out and tense) and 1-5-point Likert response range ('not at all' to 'extremely so') were used (West and Hajek 2004).

\section{Quality of life}

Data was collected on health-related quality of life using the three-level European Quality of Life-5 Dimension questionnaire (EQ-5D-3L; Prieto and Sacristan 2004). In addition, a binary variable 'presence of a mental health condition' indicating the presence/absence of mental health issues was derived from the EQ-5D-3L data. An answer indicating 'some problems' or 'extreme problems' on the anxiety/depression 
dimension was considered as an indication of potential mental health issues.

\section{Subjective stress}

The 4-item perceived stress scale (PSS) measured the extent to which respondents have felt their life to be stressful during the past month using a 5-point Likert scale (Cohen et al. 1983).

\section{Alcohol consumption}

Alcohol consumption was assessed using modified questions from the Alcohol Use Disorders Identification Test (AUDIT; Allen et al. 1997): (1) How often do you have a drink containing alcohol?; (2) How many drinks containing alcohol do you have on a typical day when you are drinking?; and (3) How many drinks containing alcohol have you had in the past week? The last two questions were answered only by participants who reported that they drink alcohol at least once a month or more frequently $(N=84$; see Online Resource 1 for more details). Based on preliminary analyses, the first two AUDIT questions were combined into a new three-level alcohol consumption variable $(N=99$; non-drinkers, light/ moderate drinkers [drinking 1-6 drinks on a typical day drinking] and heavy drinkers [drinking 7 or more drinks on a typical day drinking]) and used in the analyses.

\section{Statistical analyses}

Data were described using the mean and SD, median and inter-quartile range (IQR), and proportions. Associations were investigated using correlations and linear regressions models. To facilitate the use of linear regression and to assist with interpretation of the results, although technically ordinal variables, measures of cigarette cravings were linearly rescaled to a range of 0-100 and were treated as continuous variables (Lyratzopoulos et al. 2012). All statistical analyses were performed using Stata 13, and the significance threshold was set at 0.05 in all analyses. No attempts to impute missing data were made; consequently, some analyses did not include the whole dataset $(N=95-99)$ and, where there is missing data, this is indicated in the results. All analyses described in this section are post hoc exploratory analyses. In many analyses, multiple tests of the trial outcomes were performed. Therefore, all results must be interpreted with caution.

\section{The association between physical activity and strength of urges to smoke}

To investigate a potential association between habitual levels of PA and cigarette cravings, a series of linear regression models were applied individually with minutes of MVPA and total EE as predictors of SOU. The likelihood ratio (LR) test was used to compare the fit of nested models to assess whether the model including moderate intensity PA could be improved.

\section{Additional predictors of strength of urges to smoke}

The associations between potential predictors and SOU were investigated using a series of univariable linear regression models. Each univariable model was then repeated with adjustment for PA. All individually significant additional predictors (after adjusting for PA) identified in the individual regression models were combined in one model. All variables that were significantly associated with SOU in the individual regression models were also combined in a backward stepwise regression model. In addition, all individually significant variables were included in a backward stepwise regression model with the moderate intensity PA variable included as a predictor regardless of its contribution to the model. These models aimed to identify additional predictors of cigarette cravings, with and without adjustment for PA.

\section{Moderators of the association between physical activity and strength of urge}

The analyses of individually significant predictors of SOU (after adjusting for PA) were extended by including interaction terms with PA. Individual models (i.e. each including only one potential moderator) with the potential moderator, PA and potential moderator/PA interaction were applied to the data. Only variables demonstrating a significant interaction with PA were considered to be moderating the effects of habitual PA on cigarette cravings (Kraemer et al. 2002). If appropriate, all moderators identified in the individual regression models were combined in one model to identify all significant moderators of the relationship between PA and cravings. The LR test was used to compare the fit of nested models. We also investigated any potential moderation of the effects of PA by variables found not to be individually associated with cigarette cravings. Finally, in order to identify the most appropriate model, all significant predictors and moderators were combined. The LR test was used to compare the fit of nested models.

\section{Results}

Participants' characteristics are summarised in Table 1.

\section{The association between physical activity and strength of urge}

Table 2 summarises the association between self-reported PA (minutes of daily light, moderate, vigorous and MVPA intensities and EE) and SOU. All levels of habitual PA, except 
Table 1 Participants characteristics $(N=99)$
Table 2 Strength of urge; results of series of linear regression models including each physical activity variable individually $(N=99)$

\begin{tabular}{|c|c|c|}
\hline \multicolumn{2}{|l|}{ Variables } & Mean (SD); median [IQR] or $n / N \%$ \\
\hline \multicolumn{2}{|c|}{ Strength of urge ( $0-5$ scale) } & $2.7(1.2) ; 3.0[2,3]$ \\
\hline \multicolumn{2}{|c|}{ Minutes of light PA per day } & 992.4 (139.4); 1020 [908.6, 1088.6] \\
\hline \multicolumn{2}{|c|}{ Minutes of moderate PA per day ${ }^{a}$} & $70.3(88.5) ; 45[17.1,77.1]$ \\
\hline \multicolumn{2}{|c|}{ Minutes of vigorous PA per day } & $2.8(14.1) ; 0[0,0]$ \\
\hline \multicolumn{2}{|c|}{ Minutes of MVPA per day ${ }^{a}$} & $73.1(91.1) ; 45[17.1,77.1]$ \\
\hline \multicolumn{2}{|c|}{ Daily energy expenditure $(\mathrm{kcal} / \mathrm{kg})^{\mathrm{b}}$} & $36.05(3.9) ; 34.9[33.7,36.8]$ \\
\hline \multicolumn{2}{|l|}{ Age (years) } & $46.6(11.3) ; 47.5[38.3,55.4]$ \\
\hline \multicolumn{2}{|l|}{ EQ-5D-3L ${ }^{\mathrm{c}}$} & $0.749(0.275) ; 0.796[0.725,1]$ \\
\hline \multicolumn{2}{|l|}{ PSS (0-16 score range) } & $5.7(4.1) ; 4[2,9]$ \\
\hline \multicolumn{2}{|l|}{ MPSS (1-5 score range) } & $2.5(0.9) ; 2.3[1.8,3.1]$ \\
\hline \multicolumn{2}{|c|}{ Cigarettes smoked per day } & $21.6(14.3) ; 19.1[14.4,24.4]$ \\
\hline \multicolumn{2}{|l|}{ FTCD (0-10 score range) } & $5.6(2.0) ; 6.0[4.0,7.0]$ \\
\hline \multicolumn{2}{|c|}{ Age started smoking (years) } & $14.7(3.5) ; 14.0[13.0,16.0]$ \\
\hline \multicolumn{2}{|c|}{ mCEQ satisfaction (1-7 score range) } & $3.8(.5) ; 3.7[2.7,4.7]$ \\
\hline \multicolumn{2}{|c|}{ mCEQ reward (1-7 score range) } & $3.3(1.2) ; 3.2[2.6,4.2]$ \\
\hline \multirow[t]{3}{*}{ Alcohol consumption: $^{\mathrm{d}}$} & Non-drinkers: & $15 / 99(15)$ \\
\hline & Light/moderate drinkers: & $60 / 99(60)$ \\
\hline & Heavy drinkers: & $24 / 99(24)$ \\
\hline \multicolumn{2}{|l|}{ Gender male: } & $43 / 99(43)$ \\
\hline \multicolumn{2}{|c|}{ Presence of mental health condition ${ }^{\mathrm{e}}$} & $41 / 99(41)$ \\
\hline \multicolumn{2}{|l|}{ Employed } & $54 / 99(55)$ \\
\hline \multicolumn{2}{|l|}{ Meeting PA guidelines ${ }^{\mathrm{a}, \mathrm{f}}$} & 68/98 (70) \\
\hline
\end{tabular}

Key: EQ-5D-3L, three-level European Quality of Life-5 Dimension questionnaire, FTCD Fagerström Test for Cigarette Dependence, IQR inter-quartile range, $m C E Q$ modified Cigarette Evaluation Questionnaire, MET Metabolic equivalent of Task, MPSS mood and physical symptoms scale, MVPA Moderate and Vigorous Physical Activity, $N$ Number of participants, $P S S$ perceived stress scale, $S D$ standard deviation

${ }^{\mathrm{a}} N=98$

${ }^{\mathrm{b}} N=95$

${ }^{\mathrm{c}}$ Score of 1 represents full health, zero represents death, while negative score represents states worse than death

d Three-level 'Alcohol consumption' variable combined from the first two AUDIT questions;'non-drinkers' (no alcohol consumption), 'light/moderate drinkers' (consuming between 1 and 6 alcoholic drinks on a typical day) and 'heavy drinkers' (consumed 7 or more alcoholic drinks on a typical day). For the results of the three modified AUDIT questions see Online Resource 1

e Answered 'moderately' or 'extremely' anxious or depressed to item 5 of the EQ-5D-3L questionnaire

${ }^{\mathrm{f}}$ Participants were categorised as meeting the PA guidelines (exercising 150 or more minutes of MVPA per week) and not meeting the PA guidelines (exercising less than 30 or more minutes of MVPA per week)

\begin{tabular}{|c|c|c|c|}
\hline PA & SOU; mean difference $(95 \% \mathrm{CI})$ & $F$ statistics $(p)$ & $R^{2}(\%)$ \\
\hline Minutes of light PA per day ${ }^{a}$ & $0.053(0.02 ; 0.09)$ & $F_{(1,93)}=9.80(0.002)$ & 8.6 \\
\hline Minutes of moderate PA per day ${ }^{b}$ & $-0.082(-0.13 ;-0.03)$ & $F_{(1,96)}=10.04(0.002)$ & 8.5 \\
\hline Minutes of vigorous PA per day & $-0.054(-0.39 ; 0.28)$ & $F_{(1,97)}=0.10(0.746)$ & -0.9 \\
\hline Minutes of MVPA per day ${ }^{b}$ & $-0.078(-0.13 ;-0.03)$ & $F_{(1,96)}=9.79(0.002)$ & 8.3 \\
\hline $\begin{array}{l}\text { Daily energy expenditure } \\
(\mathrm{kcal} / \mathrm{kg})\end{array}$ & $-1.586(-2.77 ;-0.40)$ & $F_{(1,93)}=7.09(0.009)$ & 6.1 \\
\hline
\end{tabular}

Statistically significant results are shown in italics

Key: $95 \%$ CI $95 \%$ confidence interval, $M V P A$ moderate and vigorous physical activity, $N$ number of participants, $P A$ physical activity, $R^{2}$ adjusted $R^{2}, S O U$ strength of urge

${ }^{\mathrm{a}} N=95$

${ }^{\mathrm{b}} N=98$; strength of urge was linearly rescaled to $0-100$ scale 
vigorous intensity, predicted SOU $(p<0.05)$. Light intensity PA (including sedentary time) was positively associated with SOU, whereas daily EE, moderate and MVPA intensities were negatively associated with SOU. Therefore, for an increase in moderate PA of 30 min per day (within the range of the observed data), a mean reduction in SOU of around 2.5 points would be expected (based on results in Table 2). Because only eight participants reported exercising at vigorous intensity PA, the results for moderate and MVPA intensities were very similar.

We then investigated whether the addition of other PA variables to the univariate model including moderate intensity PA would improve the model fit (MVPA was not included because of collinearity with moderate intensity PA). Based on LR tests, the addition of neither light intensity PA nor EE to a model including moderate intensity PA alone significantly improved the model fit. Therefore, in all subsequent models, only moderate intensity PA was included as a measure of habitual PA.

\section{Additional predictors of strength of urge}

Categorical variables (alcohol consumption, gender, employment, meeting PA guidelines, presence of a mental health condition), and continuous variables (EQ-5D-3L, MPSS, FTCD, mCEQ reward), significantly correlated with SOU, or approaching significance (PSS ( $[p=0.064]$; for the correlations see Online Resource 2) were included in series of linear regression models investigating the effects of each potential predictor individually on SOU. PSS, MPSS, FTCD, mCEQ reward, alcohol consumption and presence of a mental health condition were found to be individually significantly associated with SOU (see Online Resource 3). The same six variables, PSS, MPSS, FTCD, mCEQ reward, alcohol consumption, and presence of a mental health condition, remained significantly associated with SOU after adjusting for moderate intensity PA (Table 3). In addition, moderate intensity PA remained significant when included in all models with one additional significant variable.

When all individually significant predictors (PSS, MPSS, FTCD, mCEQ reward, alcohol consumption, presence of a mental health condition) and moderate PA were included in the same regression model with SOU, only moderate intensity PA, MPSS and alcohol consumption remained significant with SOU $(p<0.05)$. When the backward stepwise regression model was applied (using the same variables), moderate intensity PA, MPSS and alcohol consumption were included in the model (Table 4). Repeating the backward stepwise regression model with enforced inclusion of moderate intensity PA resulted in the same model. In summary, MPSS and alcohol consumption were found to be additional predictors (adjusted for moderate intensity PA) of SOU in this population (Table 4). Both an increase in moderate intensity PA and higher alcohol consumption were associated with lower SOU; the decrease in SOU associated with alcohol consumption appeared to be driven by lower SOU among light/moderate drinkers compared with non-drinkers (mean difference $-23.46,95 \% \mathrm{CI}-34.66$ to -12.26 ). A higher MPSS score was associated with higher SOU.

\section{Moderators of the effect of moderate intensity physical activity on strength of urge}

Each potential moderator and its interaction with PA were included in an individual model with PA; gender, presence of a mental health condition, EQ-5D-3L, PSS, MPSS, cigarettes smoked per day, FTCD, age started smoking, mCEQ reward, mCEQ satisfaction, alcohol consumption, employment status and meeting PA guidelines were considered. Only significant moderators are reported. Alcohol consumption was found to moderate the relationship between moderate intensity PA and SOU. The interaction term between alcohol consumption and minutes of moderate PA (a continuous variable) was significantly different from zero $\left(F_{(2,92)}=5.51, p=0.006\right)$ overall (Table 5).

It appears that the 'non-drinkers' subgroup reported higher cigarette cravings compared with 'light/moderate drinkers' and 'heavy drinkers'; the global $F$ statistic of the three levels alcohol consumption was statistically significant (Table 5). However, the moderating effects of alcohol consumption on the relationship between moderate intensity PA and SOU suggest that both the 'non-drinkers' and 'light/moderate drinkers' are associated with lower cigarette cravings as minutes of moderate PA increases, whereas 'heavy drinkers' are associated with higher cravings as minutes of moderate PA increases (Fig. 1).

\section{Combining additional predictors of strength of urge and moderators of the effect of moderate intensity physical activity on strength of urge}

All significant predictors and moderators were analysed together to identify the most appropriate model. MPSS and alcohol consumption were identified as additional predictors of the effects of PA on SOU; alcohol consumption was also identified as a moderator of the effects of PA on SOU. The LR test was used to compare the fit of two models, the first including MPSS, PA, alcohol consumption and the interaction between PA and alcohol consumption and the second excluding the interaction term.

The LR test demonstrated that adding the interaction between PA and alcohol consumption improved the model; $\mathrm{LR}=8.12, p=0.0173$. Thus, the most appropriate 
Table 3 Series of linear regression models investigating the associations of each potential additional predictor individually on strength of urge (adjusted for moderate intensity physical activity, $N=98$ )

\begin{tabular}{|c|c|c|c|c|c|}
\hline \multirow[b]{2}{*}{ Additional predictors } & & \multicolumn{2}{|c|}{$\begin{array}{l}\text { Additional predictors } \\
\text { (as specified in the first column) }\end{array}$} & \multicolumn{2}{|l|}{ Moderate PA } \\
\hline & & $\begin{array}{l}\text { SOU, mean difference } \\
\quad(95 \% \mathrm{CI})\end{array}$ & $t$ statistics $(p)$ & $\begin{array}{l}\text { SOU, mean difference } \\
\quad(95 \% \mathrm{CI})\end{array}$ & $t$ statistics $(p)$ \\
\hline EQ-5D-3L & & $-10.23(-26.87 ; 6.41)$ & $-1.22(0.225)$ & $-0.08(-0.13 ;-0.02)$ & $-2.89(0.005)$ \\
\hline PSS & & $1.09(0.01 ; 2.18)$ & $2.01(0.048)$ & $-0.08(-0.13 ;-0.03)$ & $-3.02(0.003)$ \\
\hline MPSS & & $8.16(3.28 ; 13.04)$ & $3.32(0.009)$ & $-0.08(-0.12 ;-0.03)$ & $-3.06(0.003)$ \\
\hline FTCD & & $3.21(1.06 ; 5.36)$ & $2.96(0.004)$ & $-0.07(-0.12 ;-0.02)$ & $-2.81(0.006)$ \\
\hline mCEQ reward & & 5.51 (1.98; 9.03) & $3.10(0.003)$ & $-0.08(-0.13 ;-0.03)$ & $-3.29(0.001)$ \\
\hline 'Alcohol consumption'a & $\begin{array}{l}\text { Light/moderate } \\
\text { drinkers } \\
\text { Heavy drinkers }\end{array}$ & $\begin{array}{l}-25.36(-37.08 ;-13.62) \\
-10.19(-23.51 ; 3.14)\end{array}$ & $\begin{array}{l}\text { Global statistic }^{\mathrm{b}}: \\
\quad F_{(2,94)}=11.43, p<0.001\end{array}$ & $-0.077(-0.12 ;-0.03)$ & $-3.22(0.002)$ \\
\hline Employment status & & $5.89(-3.40 ; 15.18)$ & $1.26(0.211)$ & $-0.07(-0.13 ;-0.02)$ & $-2.80(0.006)$ \\
\hline $\begin{array}{l}\text { Presence of a mental } \\
\text { health condition }\end{array}$ & & $10.80(1.691 ; 19.91)$ & $2.32(0.021)$ & $-0.07(-0.12 ; 0.02)$ & $-2.77(0.007)$ \\
\hline Met PA guidelines & & $2.36(-8.91 ; 13.63)$ & $0.42(0.678)$ & $-0.09(-0.15 ;-0.03)$ & $-2.95(0.004)$ \\
\hline Gender & & $1.49(-7.75 ; 10.73)$ & $0.32(0.750)$ & $-0.08(-0.13 ;-0.03)$ & $-3.06(0.003)$ \\
\hline
\end{tabular}

Statistically significant results are shown in italics

Key: $E Q-5 D-3 L$ three-level European Quality of Life-5 Dimension questionnaire, $F T C D$ Fagerström Test for Cigarette Dependence, $m C E Q$ modified Cigarette Evaluation Questionnaire, MPSS mood and physical symptoms scale, $N$ number of participants, $P A$ physical activity, $P S S$ perceived stress scale, SOU strength of urge, $5 \%$ CI $95 \%$ confidence interval

a 'Non-drinkers' (no alcohol consumption), 'light/moderate drinkers' (consuming between 1 and 6 alcoholic drinks on a typical day) and 'heavy drinkers' (consumed 7 or more alcoholic drinks on a typical day)

${ }^{\mathrm{b}} p$ values for the global $F$ statistic of the three levels alcohol consumption are derived from a Wald test

'Answered 'moderately' or 'extremely' anxious or depressed to item 5 of the EQ-5D-3L questionnaire; 'not drinking alcohol' was the baseline category for 'alcohol consumption'; 'male' was the baseline category for gender; 'employed' was the baseline category for employment status; 'not meeting PA guidelines' was the baseline category for Met PA guidelines; 'lack of anxiety' was the baseline category for presence of a mental health condition

model predicting SOU in the whole population includes PA, MPSS, alcohol consumption, and the interaction between PA and alcohol consumption (Table 6); again, an increase in moderate intensity PA and higher alcohol consumption were associated with lower SOU, while a higher MPSS score was associated with higher SOU.

Table 4 Stepwise regression model showing additional predictors of strength of urge to smoke $(N=98)$

\begin{tabular}{|c|c|c|c|}
\hline & & Mean difference $(95 \% \mathrm{CI})$ & $t$ statistics $(p)$ \\
\hline $\begin{array}{l}\text { Moderate intensity PA } \\
\text { (minutes per day) }\end{array}$ & & $-0.07(-0.11 ;-0.03)$ & $-3.14(0.002)$ \\
\hline Alcohol consumption $^{a}$ & $\begin{array}{l}\text { Light/moderate } \\
\text { drinkers } \\
\text { Heavy drinkers }\end{array}$ & $\begin{array}{l}-23.46(-34.66 ;-12.26) \\
-8.07(-20.81 ; 4.66)\end{array}$ & $\begin{array}{l}\text { Global statistic }^{\mathrm{b}}: \\
\quad F_{(2,93)}=11.41, p<0.001\end{array}$ \\
\hline MPSS & & $7.42(2.97 ; 11.86)$ & $3.31(0.001)$ \\
\hline$F$ statistic & & $F_{(4,93)}=12.45 ; p<0.001$ & \\
\hline$R^{2}$ & & 0.349 & \\
\hline
\end{tabular}

Statistically significant results are shown in italics

Key: $95 \%$ CI $95 \%$ confidence interval, $P A$ physical activity

a 'Non-drinkers' (no alcohol consumption), 'light/moderate drinkers' (consuming between 1 and 6 alcoholic drinks on a typical day) and 'heavy drinkers' (consumed 7 or more alcoholic drinks on a typical day); moderate PA, PSS, MPSS, FTCD, mCEQ reward, alcohol consumption and presence of a mental health condition were included in the regression model; repeating the backward stepwise regression model without the moderate intensity PA variable kept in resulted in the same model; 'non-drinkers' was the baseline category for alcohol consumption

${ }^{\mathrm{b}} p$ values for the global $F$ statistic of the three levels alcohol consumption are derived from a Wald test 
Table 5 Linear regression showing the three levels alcohol consumption moderator of strength of urge (adjusted for physical activity, $N=98$ )

\begin{tabular}{|c|c|c|c|}
\hline & & Mean difference $(95 \% \mathrm{CI})$ & $t$ statistics $(p)$ \\
\hline $\begin{array}{l}\text { Moderate intensity PA } \\
\text { (minutes per day) }\end{array}$ & & $-0.12(-0.24 ;-0.01)$ & $-2.13(0.035)$ \\
\hline $\begin{array}{l}\text { Three levels alcohol } \\
\text { consumption }^{\text {a }}\end{array}$ & $\begin{array}{l}\text { Light/moderate drinkers } \\
\text { Heavy drinkers }\end{array}$ & $\begin{array}{l}-25.46(-39.07 ;-11.85) \\
-22.96(-38.80 ;-7.11)\end{array}$ & $\begin{array}{l}\text { Global statistic }^{\mathrm{b}}: \\
\quad F_{(2,92)}=7.05, p=0.001\end{array}$ \\
\hline \multirow[t]{2}{*}{$\begin{array}{l}\mathrm{PA} / \text { three levels alcohol } \\
\text { consumption }\end{array}$} & $\begin{array}{l}\text { Interaction between moderate } \\
\text { intensity PA and 'light/moderate } \\
\text { drinkers' }\end{array}$ & $0.01(-0.12 ; 0.14)$ & $\begin{array}{l}\text { Global statistic }^{\mathrm{b}}: \\
\quad F_{(2,92)}=5.51, p=0.006\end{array}$ \\
\hline & $\begin{array}{l}\text { Interaction between moderate intensity } \\
\text { PA and 'heavy drinkers' }\end{array}$ & $0.19(0.04 ; 0.34)$ & \\
\hline$F$ statistic & & $F_{(5,92)}=9.90 ; p<0.001$ & \\
\hline$R^{2}$ & & 0.350 & \\
\hline
\end{tabular}

Key: $95 \%$ CI $95 \%$ confidence interval, $P A$ physical activity

a 'Non-drinkers' (no alcohol consumption), 'light/moderate drinkers' (consuming between 1 and 6 alcoholic drinks on a typical day) and 'heavy drinkers' (consumed 7 or more alcoholic drinks on a typical day)

${ }^{\mathrm{b}} p$ values for the global $F$ statistic of the three levels alcohol consumption are derived from a Wald test; 'non-drinkers' was the baseline category for drinking alcohol

\section{Discussion}

This is the first study to indicate that habitual moderate intensity PA is associated with SOU over the past week. Higher levels of moderate intensity PA (measured as average minutes per week over the preceding week) were associated with lower SOU over the same period in disadvantaged smokers not ready to quit. Both the acute studies (Haasova et al. 2014) and the current study suggest that moderate intensity PA is the strongest PA predictor of cigarette cravings; however, it must be highlighted that the design and population of the studies varied significantly. The effects of habitual moderate PA appeared to be much smaller when compared with the acute data; an increase in habitual moderate PA of 5-40 min per day (within the range of the observed data) was associated with a mean reduction in SOU of around $0.4-3.3$ points on a 0-100 scale compared with an approximate cravings reduction of $30 \%$ associated with acute bouts of PA lasting from 5 $40 \mathrm{~min}$ (Haasova et al. 2013; 2014). A recent Cochrane review of exercise interventions for smoking cessation identified 20 RCTs comparing exercise and cessation programmes with at least 6 months follow-up (Ussher et al. 2014). However, only one study (Marcus et al. 1999) showed long term benefits of exercise on smoking cessation. Notably, most studies included in the Cochrane review did not aim to increase levels of habitual PA (Ussher et al. 2014); regular participation in PA, rather than 1-3 sessions per week, may have greater enduring effects on smoking cessation outcomes.

MPSS and alcohol consumption were identified as two additional predictors of SOU; also, participants who
Fig. 1 The relationship between Alcohol consumption and strength of urge in the whole population $(N=98)$. Notes: $P A$ physical a, SOU strength of urge

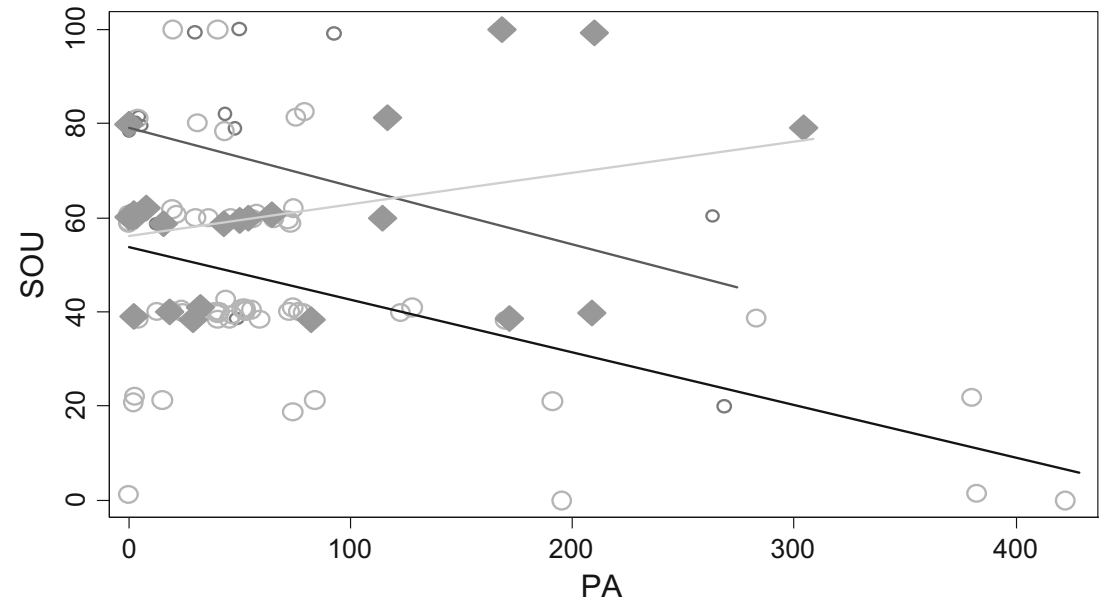

\begin{tabular}{|lll|}
\hline$\circ \quad$ No alcohol consumption & & Light/moderate drinkers \\
Heavy drinkers & No alcohol consumption \\
& Light/moderate drinkers & Heavy drinkers \\
\hline
\end{tabular}


Table 6 Strength of urge; the most appropriate model in the whole population

\begin{tabular}{|c|c|c|c|}
\hline & & Mean difference $(95 \% \mathrm{CI})$ & $t$ statistics $(p)$ \\
\hline $\begin{array}{l}\text { Moderate intensity PA } \\
\text { (minutes per day) }\end{array}$ & & $-0.12(-0.23 ;-0.01)$ & $-2.16(0.033)$ \\
\hline $\begin{array}{l}\text { Three levels alcohol } \\
\text { consumption }^{\text {a }}\end{array}$ & $\begin{array}{l}\text { Light/moderate drinkers } \\
\text { Heavy drinkers }\end{array}$ & $\begin{array}{l}-24.64(-37.77 ;-11.46) \\
-19.53(-35.05 ;-4.07)\end{array}$ & $\begin{array}{l}\text { Global statistic }^{\mathrm{b}}: \\
\quad F_{(2,91)}=6.91, p=0.002\end{array}$ \\
\hline $\begin{array}{l}\mathrm{PA} / \text { three levels alcohol } \\
\text { consumption }\end{array}$ & $\begin{array}{l}\text { Interaction between moderate } \\
\text { intensity PA and 'light/moderate } \\
\text { drinkers' } \\
\text { Interaction between moderate } \\
\text { intensity PA and 'heavy drinkers' }\end{array}$ & $0.67(0.02 ; 0.31)$ & $\begin{array}{l}\text { Global statistic }^{\mathrm{b}}: \\
\quad F_{(2,91)}=3.93, p=0.023\end{array}$ \\
\hline MPSS & & $6.18(1.76 ; 10.59)$ & $2.78(0.007)$ \\
\hline$F$ statistic & & $F_{(6,91)}=10.13 ; p<0.001$ & \\
\hline$R^{2}$ & & 0.309 & \\
\hline
\end{tabular}

Key: $95 \%$ CI $95 \%$ confidence interval, PA physical activity, MPSS mood and physical symptoms scale

a 'Non-drinkers' (no alcohol consumption), 'light/moderate drinkers' (consuming between 1 and 6 alcoholic drinks on a typical day) and 'heavy drinkers' (consumed 7 or more alcoholic drinks on a typical day)

${ }^{\mathrm{b}} p$ values for the global $F$ statistic of the three levels alcohol consumption are derived from a Wald test; 'non-drinkers' was the baseline category for alcohol consumption

consumed alcohol had lower cravings compared with nondrinkers. Higher alcohol consumption was associated with lower SOU, while a higher MPSS score was associated with higher SOU. The difference between participants who consumed alcohol and those who did not cannot be readily explained. Whilst the relationship between PA levels and smoking prevalence is quite distinct, the relationship between PA and alcohol is much more complicated and is rarely reported to be linear in nature as it is with smoking (French et al. 2009a; Lisha et al. 2013; Noble et al. 2015). Also, the relationship between PA and alcohol may be influenced by the type of activity (Wichstrom and Wichstrom 2009), so a complicated relationship and interactions are to be expected (Ekkekakis 2013; Ussher 2014). One possible explanation for the finding may be that participants who consumed alcohol reported lower cravings as their threshold for smoking was lower compared with participants who did not consume alcohol. Indeed, 'non-drinkers' reported higher cigarette cravings compared with 'light/moderate drinkers' and 'heavy drinkers' across all levels of PA. In addition, if an addiction is founded on reward and pleasure derived from a behaviour, then it is possible that in the present case heavy drinkers satisfy that need from drinking and hence have a lower need or urge for cigarettes. While an alternative scenario could be that high levels of alcohol consumption actually reduces inhibition to refrain from smoking when faced with high urges to smoke, our findings do not appear to support this.

Interestingly, measures of smoking dependence, such as FTCD and the number of cigarette smoked per day, were not found to predict SOU in addition to moderate habitual PA, MPSS and alcohol consumption. The association between habitual PA and trait cigarette cravings appears to be comparable for both light and heavy smokers.
Although an association between habitual PA and SOU was identified, an explanation for this link is beyond the scope of this study and requires further research. Speculatively, several mechanisms may be operating alone or in combination at different times for different people (Faulkner and Carless 2006). A new conceptual model for smoking cessation suggests that habitual PA may help to attenuate the smoking-induced decline in executive functioning impairment (Loprinzi et al. 2015a). The same authors found statistically significant associations between habitual PA and psychological outcomes in two large cross-sectional studies; smokers who were less physically active were also more likely to be depressed (Loprinzi et al. 2014), and exercise was found to facilitate smoking cessation via exercise-induced increases in smoking-specific self-efficacy in young smokers (Loprinzi et al. 2015b).

Finally, alcohol consumption was found to moderate the relationship between moderate intensity PA and SOU. The interactions revealed that for 'heavy drinkers', cravings increased as PA increased. It appeared that smokers consuming seven or more drinks per day on a typical day they drink lost the protective effect of habitual PA on SOU. For reasons which are unclear, this finding suggests that not all smokers may benefit from increasing their PA levels; however, more research is needed to replicate this effect.

Based on the results of the current study, further research exploring the association between habitual PA and cigarette cravings and other smoking cessation outcomes is needed before a role of habitual PA in smoking cessation can be established. Similarly, an investigation into the moderating effects of alcohol drinking is warranted. Habitual PA and alcohol consumption could be recommended as a routine 
measure in new smoking cessation trials. This would help to determine the role of PA and alcohol consumption in smoking cessation.

\section{Limitations}

All results presented and discussed in this paper are based on exploratory post hoc cross-sectional analyses; therefore, the direction of any associations cannot be determined. The results seems to either suggest that doing more PA will lead to lower cigarette cravings or smokers with lower cravings are likely to do more PA. However, the findings might be very different for abstinent smokers or smokers trying to quit. In addition, the analyses here relied on self-reported PA and cigarette cravings data as reported over the last week; more objective measures such as accelerometry PA data may be needed. Also, it may be that PA data collection over 7 days is not sufficiently long to measure the chronic level of PA. Similarly, multiple SOU measures over a longer period of time may be needed to assess the level of trait cravings. Finally, alcohol consumption, the moderator of the relationship between moderate intensity PA and SOU, consists only of the frequency of alcohol consumption and pattern of drinking on a typical day. This approach has many limitations including having no indication of the total amount of alcohol consumed (which can be linked to safe consumption guidelines). However, there is a general lack of a standard classification for alcohol consumption (Furtwaengler and de Visser 2013; Nutt and Rehm 2014). In summary, all results must be interpreted with caution and should be only used as an indication for future research.

\section{Conclusion}

Habitual PA was found to be significantly associated with SOU, with moderate PA being the best predictor. An increase in moderate PA was associated with lower SOU in disadvantaged smokers not ready to quit. Additional predictors of the effects of PA on SOU were identified; a higher MPSS score was associated with higher SOU, while a higher alcohol consumption was associated with a lower SOU. In addition, alcohol consumption was found to be a moderator of the relationship; for 'heavy drinkers' (defined as consuming seven or more alcoholic drinks on a typical day), increased minutes of moderate PA appeared to be associated with higher cravings. However, results of this study are exploratory and must be interpreted with caution. Further research into the moderating effects of alcohol drinking on the effects of habitual PA on cigarette cravings is needed.

Acknowledgments This paper presents independent research funded by the National Institute for Health Research (NIHR) under its Health Technology Assessment Research Programme (project number 07/78/02, published in 2014 in Health Technology Assessment journal, 18:4), and with the support of additional internal institutional funds (see the HTA Programme website (www.hta.ac.uk) for further project information). The views expressed are those of the authors and not necessarily those of the NHS, the NIHR or the Department of Health.

We would like to acknowledge the wider input of the EARS researcher team who contributed to the design and conduct of the study, and the participants, namely, Colin J Greaves, Rod S Taylor, Colin Green, Rebecca Kandiyali, Paul Aveyard, Richard Ayres, Richard Byng, John L Campbell, Susan Michie and Robert West.

\section{Compliance with ethical standards}

Declaration of interest The research was conducted with the support of internal institutional funds. The authors have received no other direct or indirect support, and none of the researchers have any connection with the tobacco or pharmaceutical industries or anybody substantially funded by one of these organisations.

Conflict of interest The authors declare that they have no conflicts of interest.

Open Access This article is distributed under the terms of the Creative Commons Attribution 4.0 International License (http:// creativecommons.org/licenses/by/4.0/), which permits unrestricted use, distribution, and reproduction in any medium, provided you give appropriate credit to the original author(s) and the source, provide a link to the Creative Commons license, and indicate if changes were made.

\section{References}

Allen JP, Litten RZ, Fertig JB, Babor T (1997) A review of research on the Alcohol Use Disorders Identification Test (AUDIT). Alcohol Clin Exp Res 21:613-619

Bellis MA, Hughes K, Nicholls J, Sheron N, Gilmore I, Jones L (2016) The alcohol harm paradox: using a national survey to explore how alcohol may disproportionately impact health in deprived individuals. BMC Public Health 16:1-10

Blair SN, Haskell WL, Ho P, Paffenbarger RS Jr, Vranizan KM, Farquhar JW, Wood PD (1985) Assessment of habitual physical activity by a seven-day recall in a community survey and controlled experiments. Am J Epidemiol 122:794-804

Bloom EL, Abrantes AM, Fokas KF, Ramsey SE, Brown RA (2012) Gender differences in the relationship between physical activity and smoking among psychiatrically hospitalized adolescents. Mental Health Physical Activity 5:136-140

Bull FC, The Expert Working Groups (2010) Physical activity guidelines in the U.K.: review and recommendations. School of Sport EaHS, Loughborough University

Cappelleri JC, Bushmakin AG, Baker CL, Merikle E, Olufade AO, Gilbert DG (2007) Confirmatory factor analyses and reliability of the modified cigarette evaluation questionnaire. Addict Behav 32: 912-923

Cohen S, Kamarck T, Mermelstein R (1983) A global measure of perceived stress. J Health Soc Behav 24:385-396

Ekkekakis P (2013) Routledge handbook of physical activity and mental health. Routledge, London

Fagerström K (2012) Determinants of tobacco use and renaming the FTND to the Fagerström Test for Cigarette Dependence. Nicotine Tobacco Res Off J Soc Res Nicotine Tobacco 14:75-78

Faulkner G, Carless D (2006) Physical activity in the process of psychiatric rehabilitation: theoretical and methodological issues. Psychiatr Rehabil J 29:258-266 
French MT, Popovici I, Maclean JC (2009) Do alcohol consumers exercise more? Findings from a national survey. Am J Health Promot 24: $2-10$

Furtwaengler NA, de Visser RO (2013) Lack of international consensus in low-risk drinking guidelines. Drug Alcohol Rev 32:11-18

Haasova M, Warren FC, Ussher M, Janse Van Rensburg K, Faulkner G, Cropley M, Byron-Daniel J, Everson-Hock ES, Oh H, Taylor AH (2013) The acute effects of physical activity on cigarette cravings: systematic review and meta-analysis with individual participant data. Addiction 108:26-37

Haasova M, Warren FC, Ussher M, Janse Van Rensburg K, Faulkner G, Cropley M, Byron-Daniel J, Everson-Hock ES, Oh H, Taylor AH (2014) The acute effects of physical activity on cigarette cravings: exploration of potential moderators, mediators and physical activity attributes using individual participant data (IPD) meta-analyses. Psychopharmacology 231:1267-1275

Heatherton T, Koslowski L, Frecker R, Fagerstrom K (1991) The Fagestrom Test for Nicotine Dependence: a revision of the Fagestrom tolerance questionnaire. Br J Addict 86:1119-1127

Kaczynski AT, Manske SR, Mannell RC, Grewal K (2008) Smoking and physical activity: a systematic review. Am J Health Behav 32:93-110

Killen JD, Fortmann SP (1997) Craving is associated with smoking relapse: findings from three prospective studies. Exp Clin Psychopharmacol 5:137-142

Korhonen T, Kujala UM, Rose RJ, Kaprio J (2009) Physical activity in adolescence as a predictor of alcohol and illicit drug use in early adulthood: a longitudinal population-based twin study. Twin Res Hum Genet 12:261-268

Kraemer HC, Wilson GT, Fairburn CG, Agras WS (2002) Mediators and moderators of treatment effects in randomized clinical trials. Arch Gen Psychiatry 59:877-883

Kwan M, Bobko S, Faulkner G, Donnelly P, Cairney J (2014) Sport participation and alcohol and illicit drug use in adolescents and young adults: a systematic review of longitudinal studies. Addict Behav 39:497-506

Liangpunsakul S, Crabb DW, Qi R (2010) Relationship among alcohol intake, body fat, and physical activity: a population-based study. Ann Epidemiol 20:670-675

Lisha NE, Sussman S (2010) Relationship of high school and college sports participation with alcohol, tobacco, and illicit drug use: a review. Addict Behav 35:399-407

Lisha NE, Sussman S, Fapa F, Leventhal AM (2013) Physical activity and alcohol use disorders. Am J Drug Alcohol Abuse 39:115-120

Loprinzi PD, Herod SM, Walker JF, Cardinal BJ, Mahoney SE, Kane C (2015a) Development of a conceptual model for smoking cessation: physical activity, neurocognition, and executive functioning. Res Q Exerc Sport 86(4):338-346

Loprinzi PD, Walker JF, Kane C, Cardinal BJ (2014) Physical activity moderates the association between nicotine dependence and depression among U.S. smokers. Am J Health Promot 29:37-42

Loprinzi PD, Wolfe CD, Walker JF (2015a) Exercise facilitates smoking cessation indirectly via improvements in smoking-specific self-efficacy: prospective cohort study among a national sample of young smokers. Prev Med 81:63-66
Lyratzopoulos G, Elliott M, Barbiere JM, Henderson A, Staetsky L, Paddison C, Campbell J, Roland M (2012) Understanding ethnic and other socio-demographic differences in patient experience of primary care: evidence from the English General Practice Patient Survey. BMJ Qual Saf 21:21-29

Marcus BH, Albrecht AE, King TK, Parisi AF, Pinto BM, Roberts M, Niaura RS, Abrams DB (1999) The efficacy of exercise as an aid for smoking cessation in women: a randomized controlled trial. Arch Intern Med 159:1229-1234

Marcus BH, Lewis BA, Hogan J, King TK, Albrecht AE, Bock B, Parisi AF, Niaura R, Abrams DB (2005) The efficacy of moderateintensity exercise as an aid for smoking cessation in women: a randomized controlled trial. Nicotine Tobacco Res Off J Soc Res Nicotine Tobacco 7:871-880

Noble N, Paul C, Turon H, Oldmeadow C (2015) Which modifiable health risk behaviours are related? A systematic review of the clustering of Smoking, Nutrition, Alcohol and Physical activity ('SNAP') health risk factors. Prev Med 81:16-41

Nutt DJ, Rehm J (2014) Doing it by numbers: a simple approach to reducing the harms of alcohol. J Psychopharmacol 28:3-7

Piasecki TM, Jorenby DE, Smith SS, Fiore MC, Baker TB (2003) Smoking withdrawal dynamics: II. Improved Tests WithdrawalRelapse Relations J Abnorm Psychol 112:14-27

Prieto L, Sacristan JA (2004) What is the value of social values? The uselessness of assessing health-related quality of life through preference measures. BMC Med Res Methodol 4:10

Reed J, Ones DS (2006) The effect of acute aerobic exercise on positive activated affect: a meta-analysis. Psychol Sport Exerc 7:477-514

Roberts V, Maddison R, Simpson C, Bullen C, Prapavessis H (2012) The acute effects of exercise on cigarette cravings, withdrawal symptoms, affect, and smoking behaviour: systematic review update and meta-analysis. Psychopharmacology 222:1-15

Taylor AH, Thompson TP, Greaves CJ, Taylor RS, Green C, Warren FC, Kandiyali R, Aveyard P, Ayres R, Byng R, Campbell JL, Ussher $\mathrm{MH}$, Michie S, West R (2014) A pilot randomised trial to assess the methods and procedures for evaluating the clinical effectiveness and cost-effectiveness of Exercise Assisted Reduction then Stop (EARS) among disadvantaged smokers. Health Technol Assess 18:1-324

Tiffany ST, Wray JM (2012) The clinical significance of drug craving. Ann N Y Acad Sci 1248:1-17

Ussher M (2014) Theory and practical applications. In: Clow A, Edmunds S (eds) Evidence for the impact of physical activity on addictive behavior. Human Kinetics, Champaign Illinois

Ussher MH, Taylor AH, Faulkner GE (2014) Exercise interventions for smoking cessation. Cochrane Database Syst Rev 8:CD002295

West R, Hajek P (2004) Evaluation of the mood and physical symptoms scale (MPSS) to assess cigarette withdrawal. Psychopharmacology 177:195-199

West RJ, Russell MAH (1985) Pre-abstinence smoke intake and smoking motivation as predictors of severity of cigarette withdrawal symptoms. Psychopharmacology 87:334-336

Wichstrom T, Wichstrom L (2009) Does sports participation during adolescence prevent later alcohol, tobacco and cannabis use? Addiction 104:138-149 